\title{
Influence of Induced Polarization on the Dynamics and Folding Free Energy of the Amyloid $\beta$-Peptide
}

\author{
Justin A. Lemkul \\ Department of Biochemistry, Virginia Tech
}

American Chemical Society National Meeting

Spring 2020 


\section{The Classical Drude Oscillator Model}

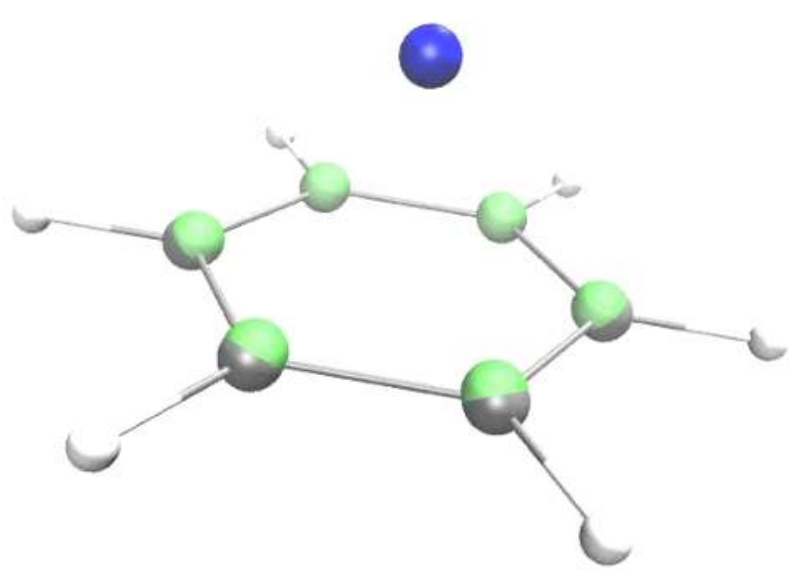

Response to electric field and molecular polarizability
A
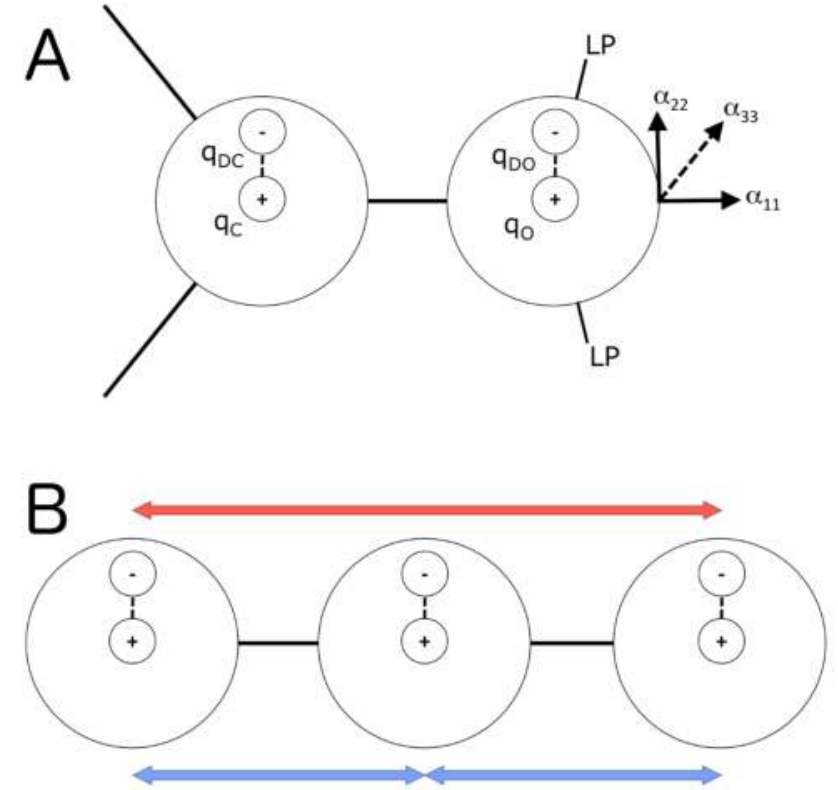

C

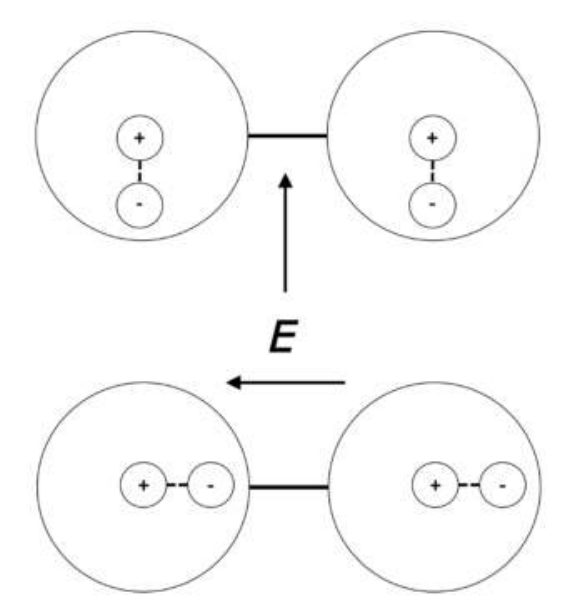

$\alpha=\frac{q_{\mathrm{D}}^{2}}{k_{\mathrm{D}}}$

Neighboring dipole screening

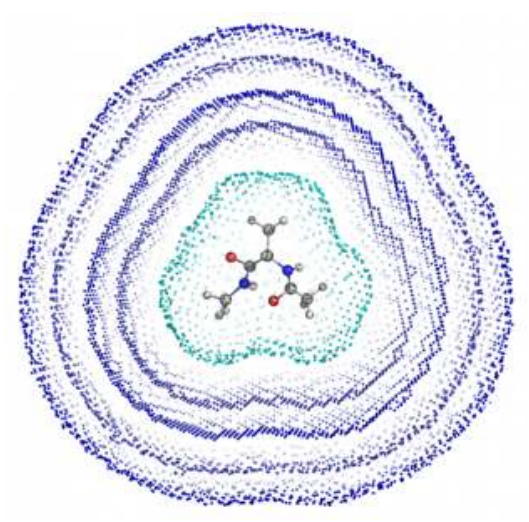




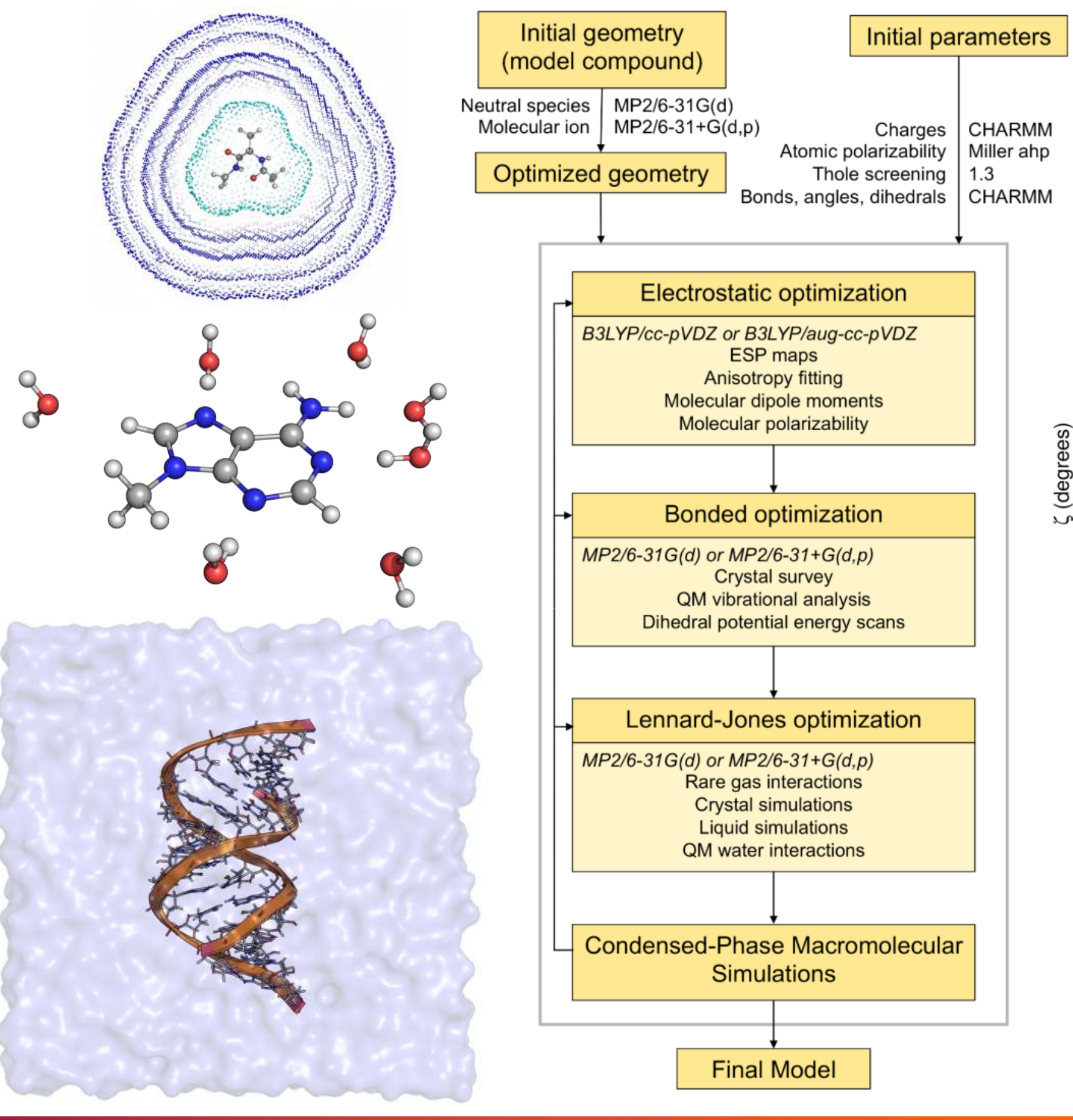

JAL, J. Huang, B. Roux, and A.D. MacKerell Jr. (2016) Chem. Rev. 116: 4983-5013.

V7 VIRGINIA 


\section{$\alpha$-Helix Cooperativity and Macrodipole}

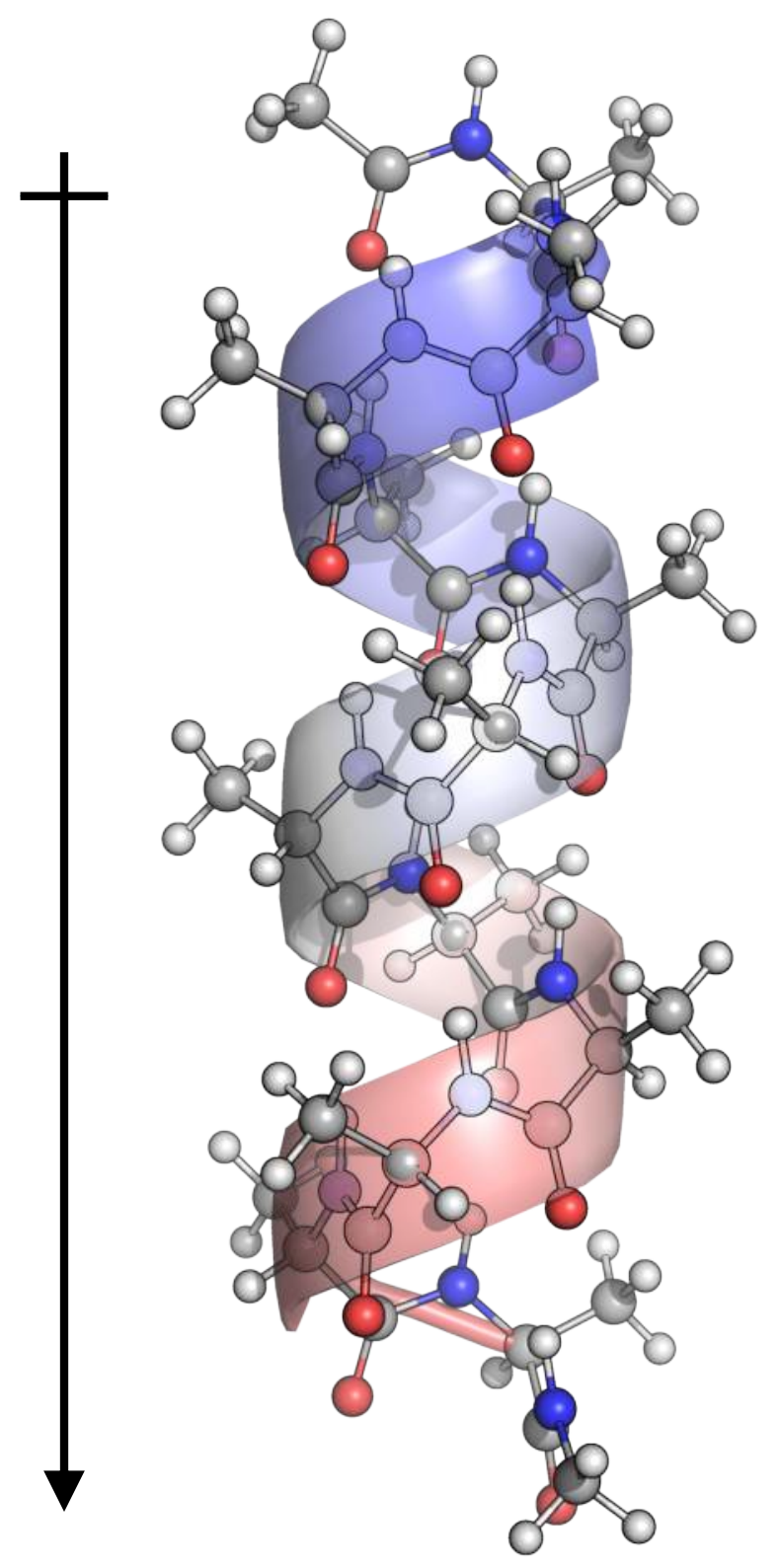

- Hol et al. (1978) Nature 273: 443-446

"Theoretical considerations indicate an increase in the peptide dipole moment by polarisation due to hydrogen bonds in the $\alpha$ helix, yielding values up to 5 $D$ [A. Wada, 1976]. In the following we have ignored this polarisation, with the possible consequences of an underestimation of electric fields."

- Polarization is an important contribution to protein folding and the overall physical properties of $\alpha$-helices

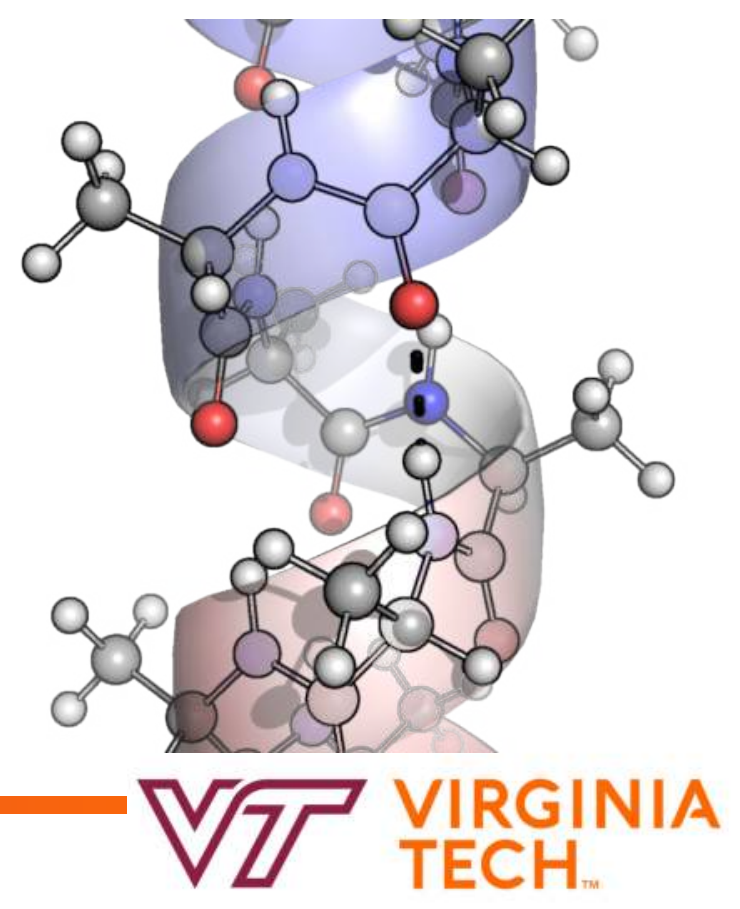


Mutations to $A \beta$

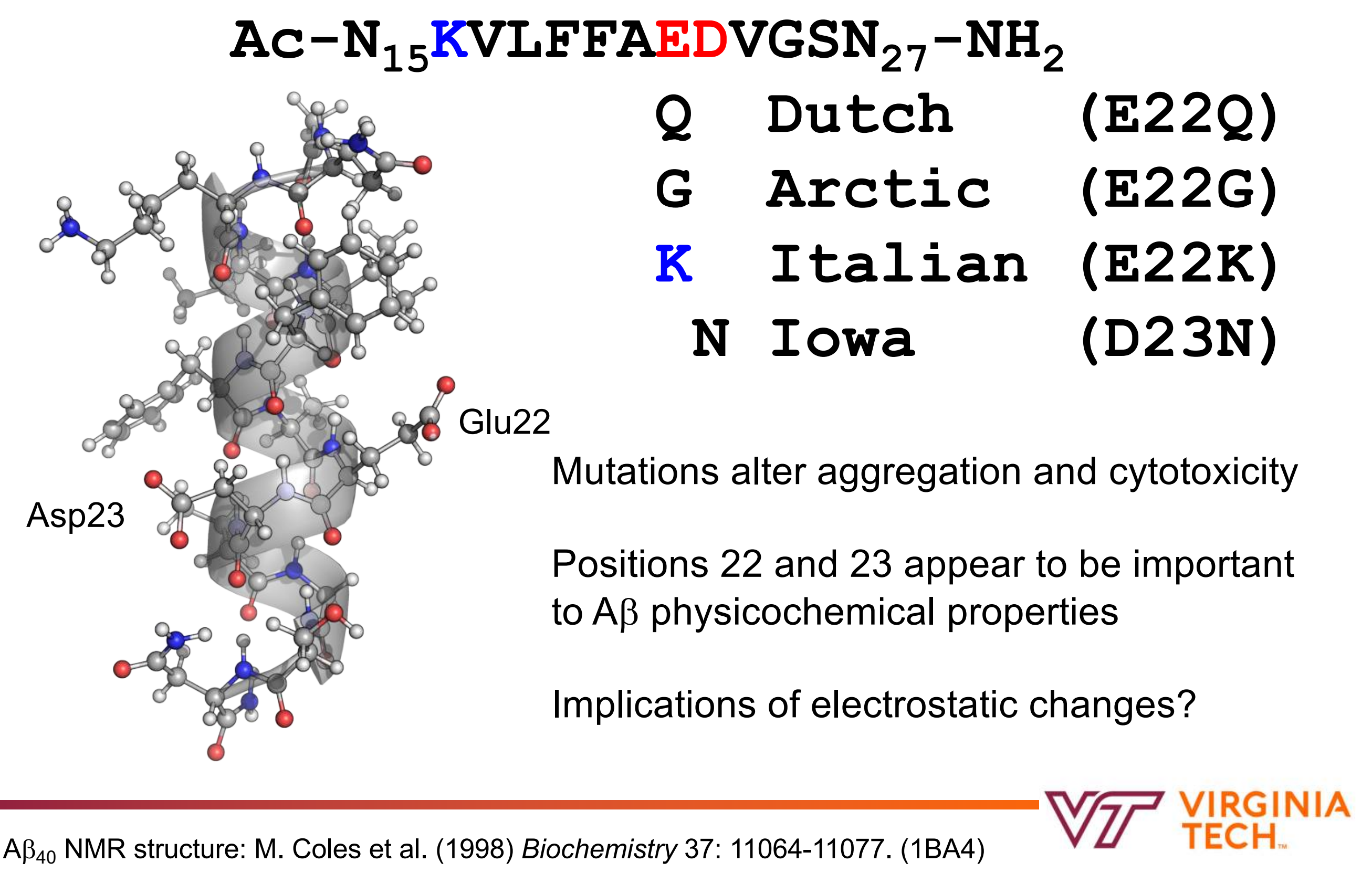




\section{WT $A \beta_{15-27}$ : Electrostatic Clash}

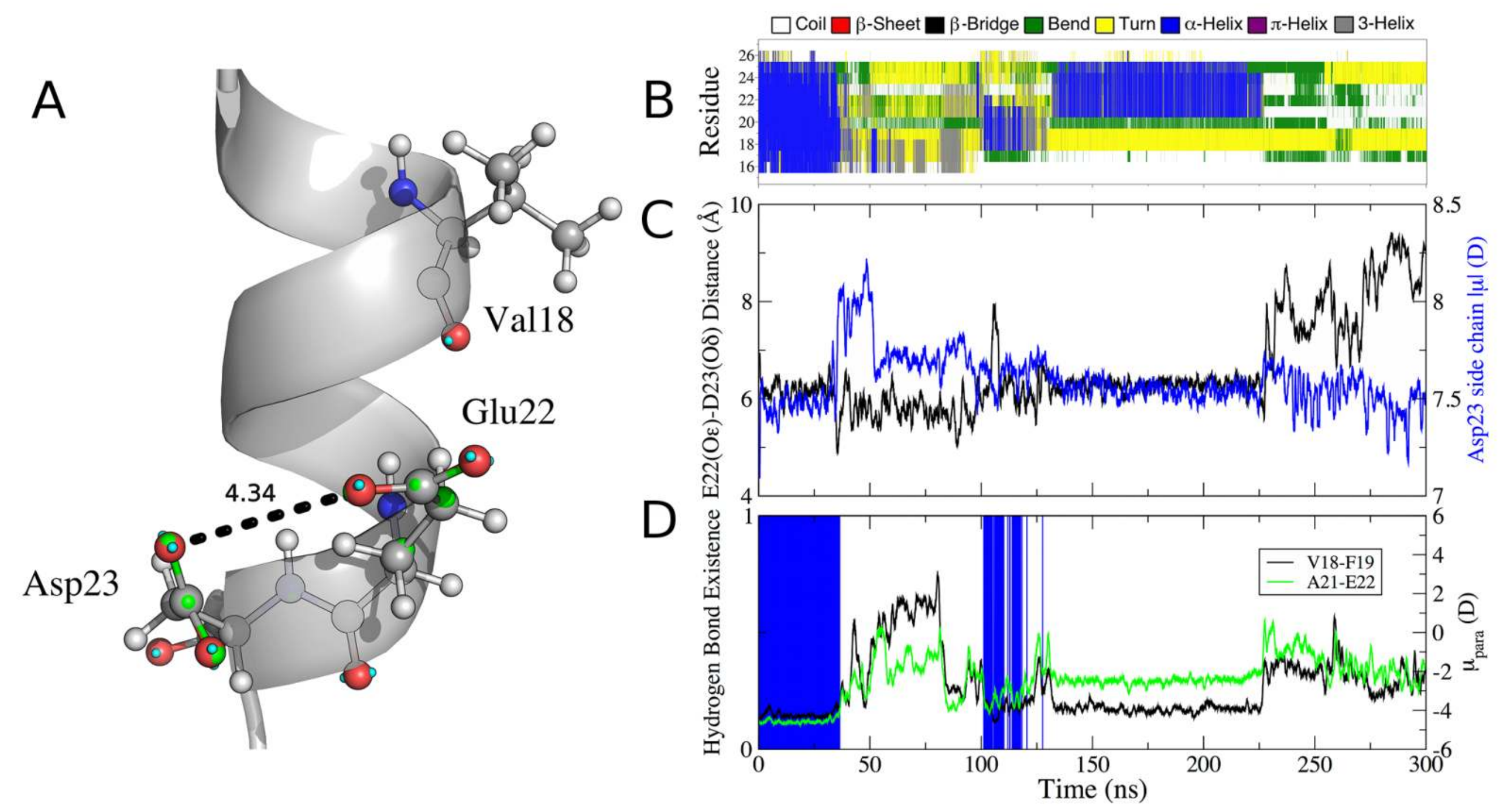

JAL, J. Huang, A.D. MacKerell Jr. (2015) J. Phys. Chem. B 119: 15574-15582. 


\section{Influence of Mutations on $A \beta \Delta G_{\text {fold }}$}
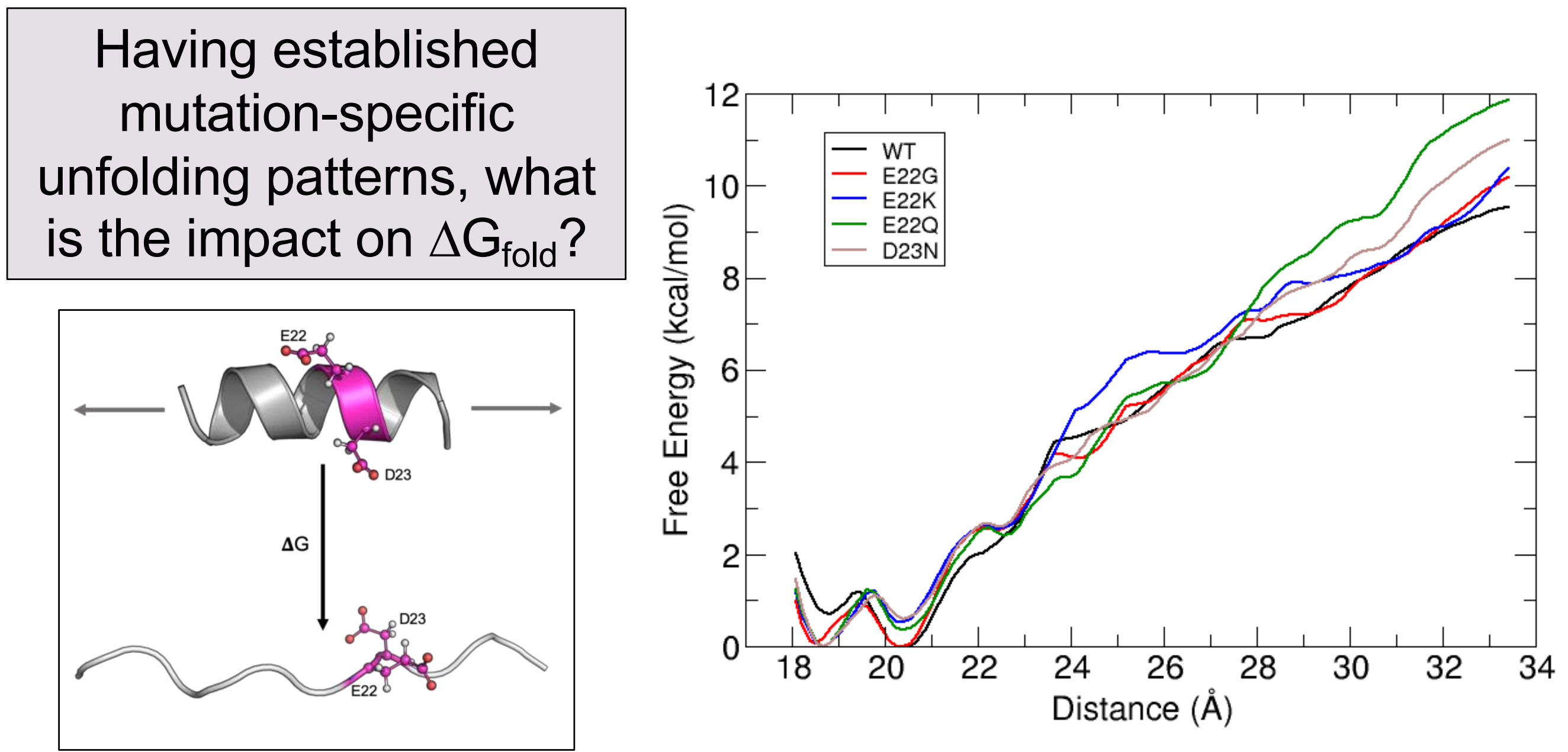

How does induced polarization impact $\Delta \mathrm{G}_{\text {fold }}$ ? 


\section{Influence of Mutations on $A \beta \Delta G_{\text {fold }}$}

$\begin{gathered}\text { Having established } \\
\text { mutation-specific } \\
\text { unfolding patterns, what } \\
\text { is the impact on } \Delta \mathrm{G}_{\text {fold }} ?\end{gathered}$
\begin{tabular}{|c|c|}
\hline & $\Delta \mathbf{G}_{\text {fold }}\left(\mathbf{k c a l ~ m o l}^{-1}\right)$ \\
\hline WT & $-4.6 \pm 0.7$ \\
\hline E22G & $-5.4 \pm 0.3$ \\
\hline E22K & $-6.1 \pm 0.6$ \\
\hline E22Q & $-5.6 \pm 0.8$ \\
\hline D23N & $-3.5 \pm 0.8$ \\
\hline
\end{tabular}

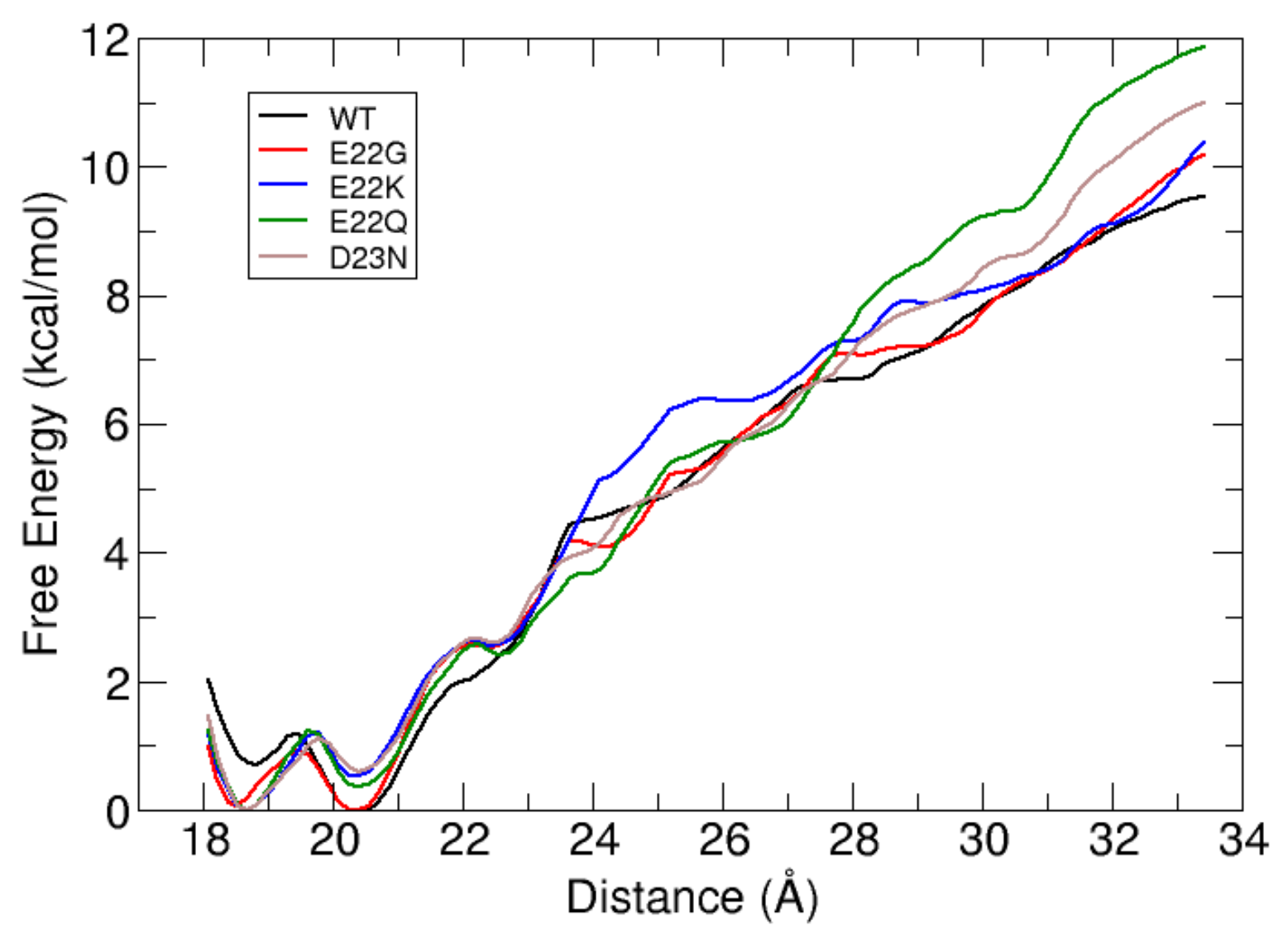

How does induced polarization impact $\Delta \mathrm{G}_{\text {fold }}$ ? 


\section{Peptide Bond Dipole Moment Enhancement}

Total dipole enhancement

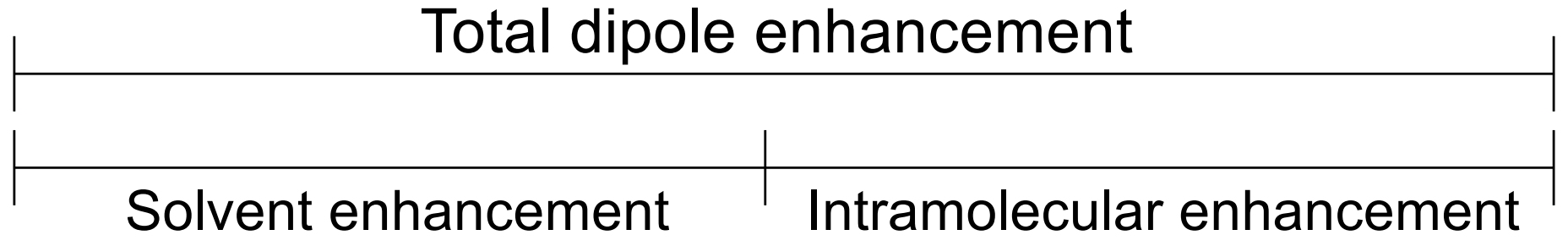

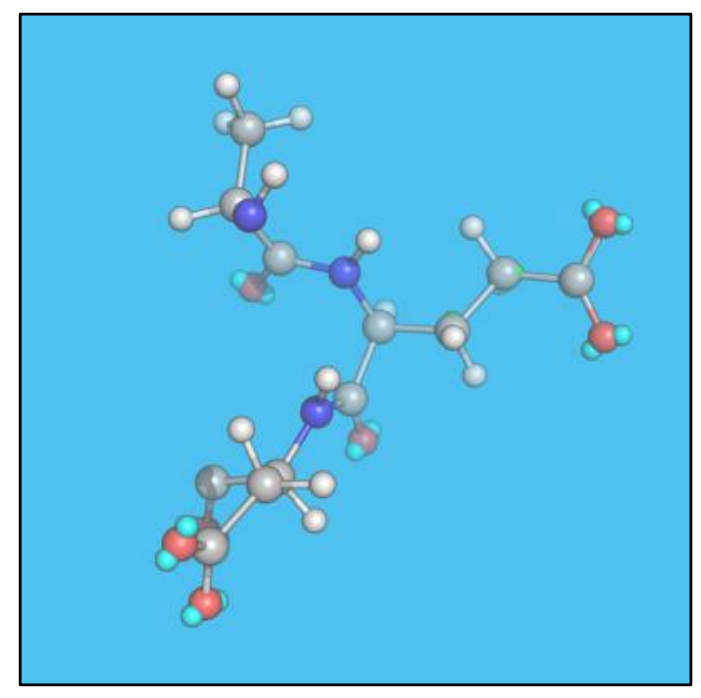

$\mu_{\text {total }}$

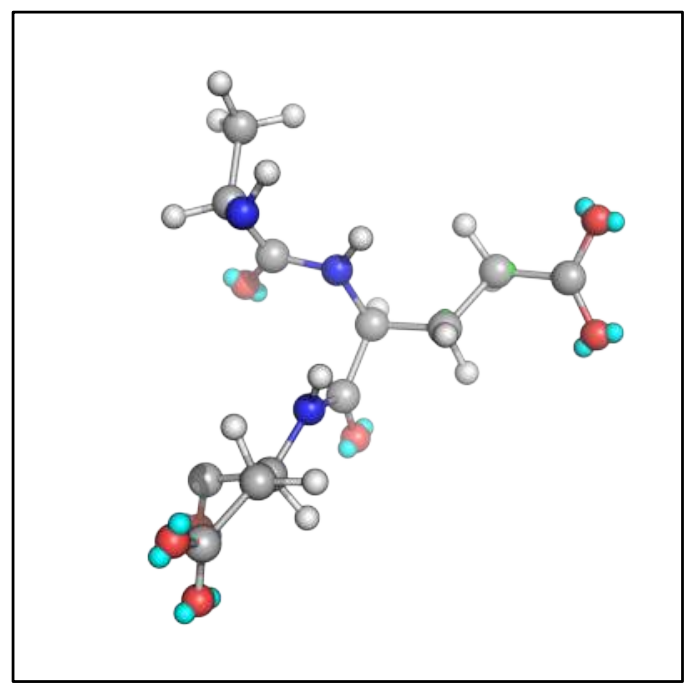

$\mu_{\text {intramolecular }}$

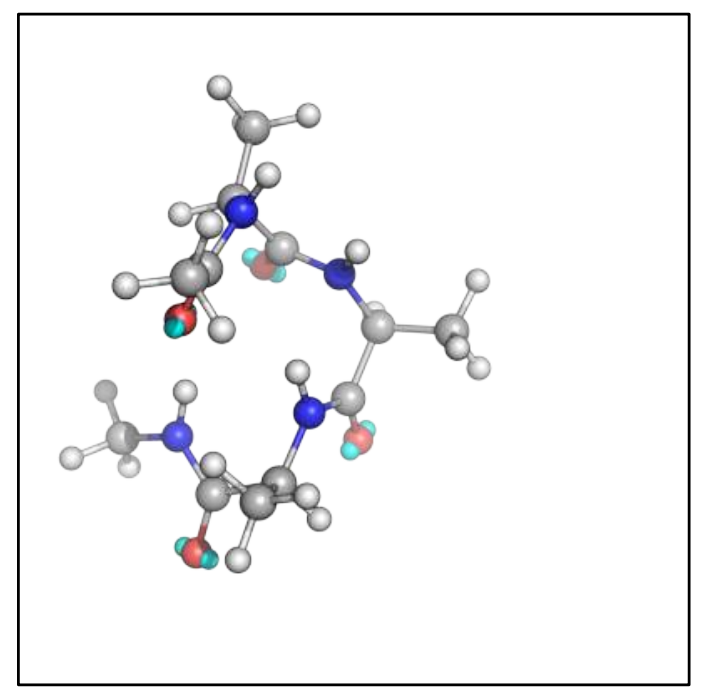

$\mu_{\text {intrinsic }}$

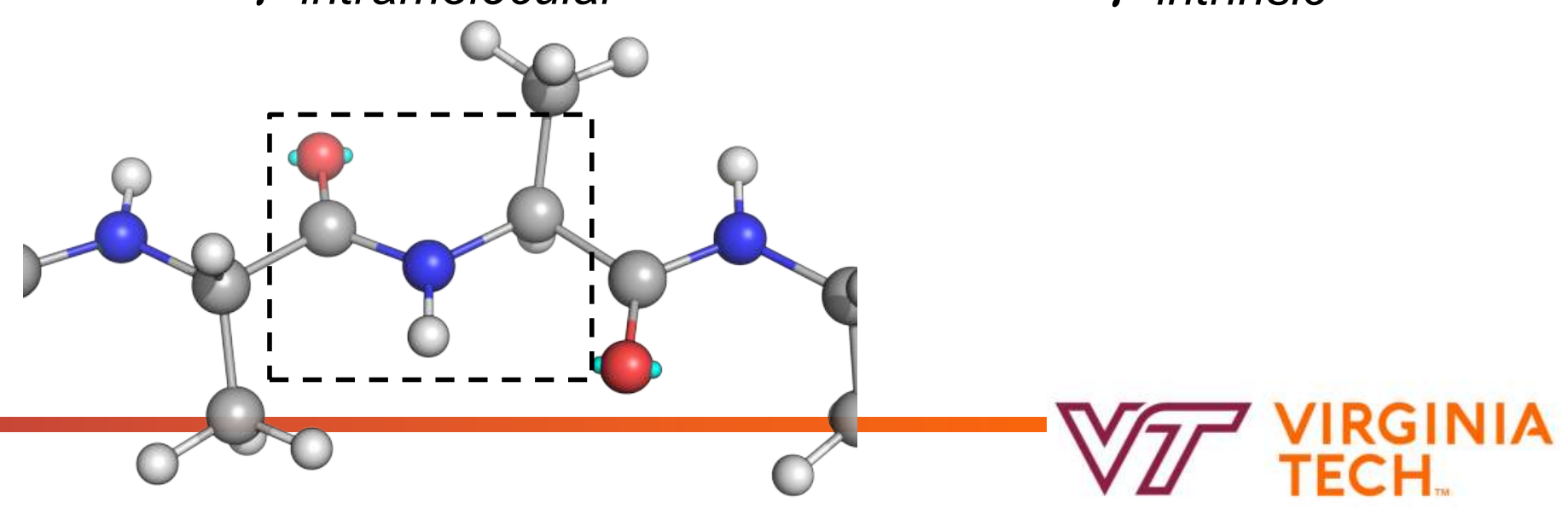




\section{Peptide Bond Dipole Moment Enhancement}

Total dipole enhancement

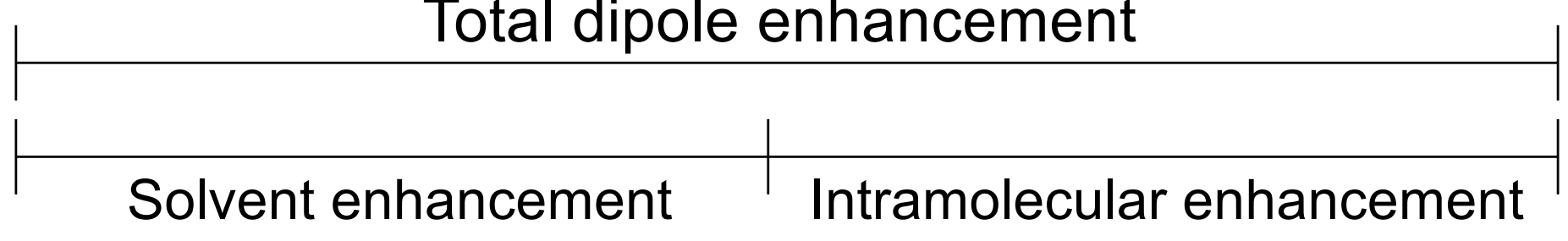

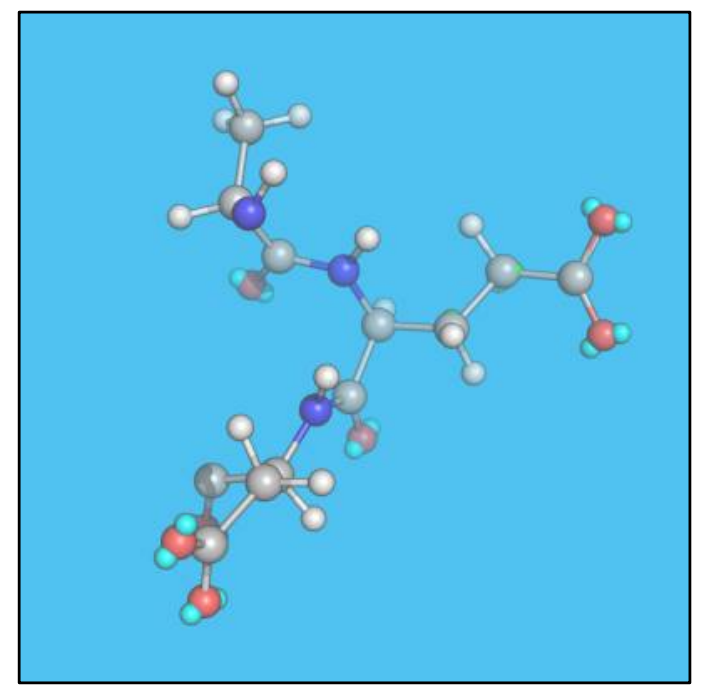

$\mu_{\text {total }}$

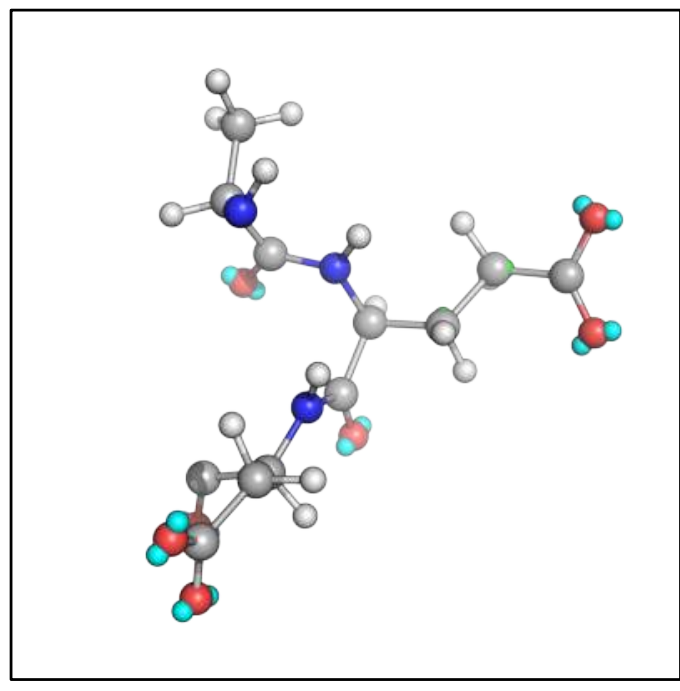

$\mu_{\text {intramolecular }}$

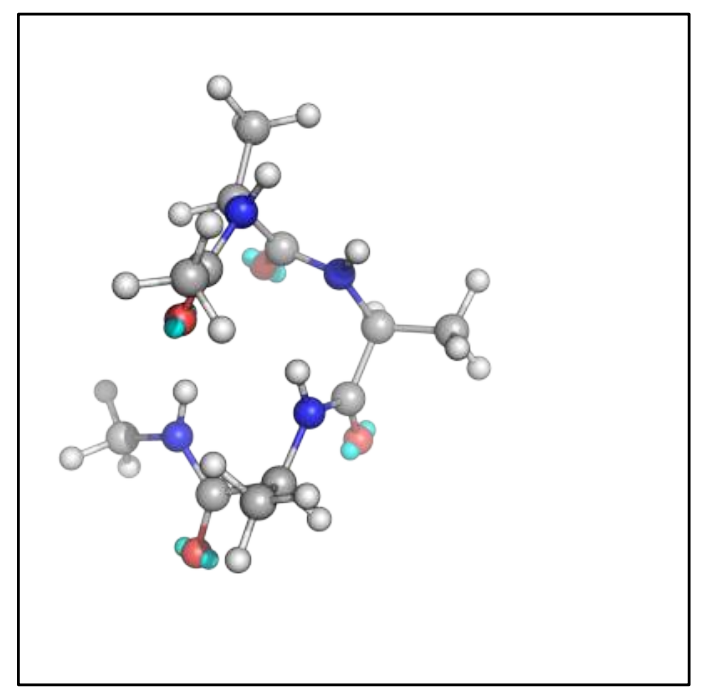

$\mu_{\text {intrinsic }}$

Remove water, relax Drudes

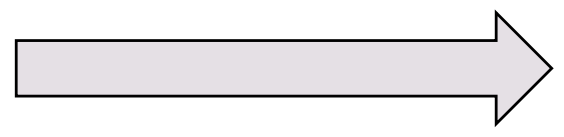

Compute contribution of water to the peptide-bond dipole moment
Build $\mathrm{Ala}_{3}$ with same $(\phi, \psi)$

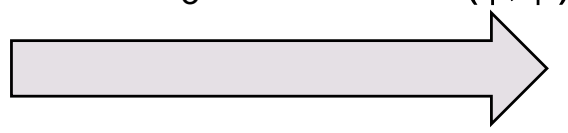

From the dipole moment that is intrinsic to a polypeptide geometry, compute contribution from side chain 


\section{Dipole Enhancement as $A \beta$ Unfolds}

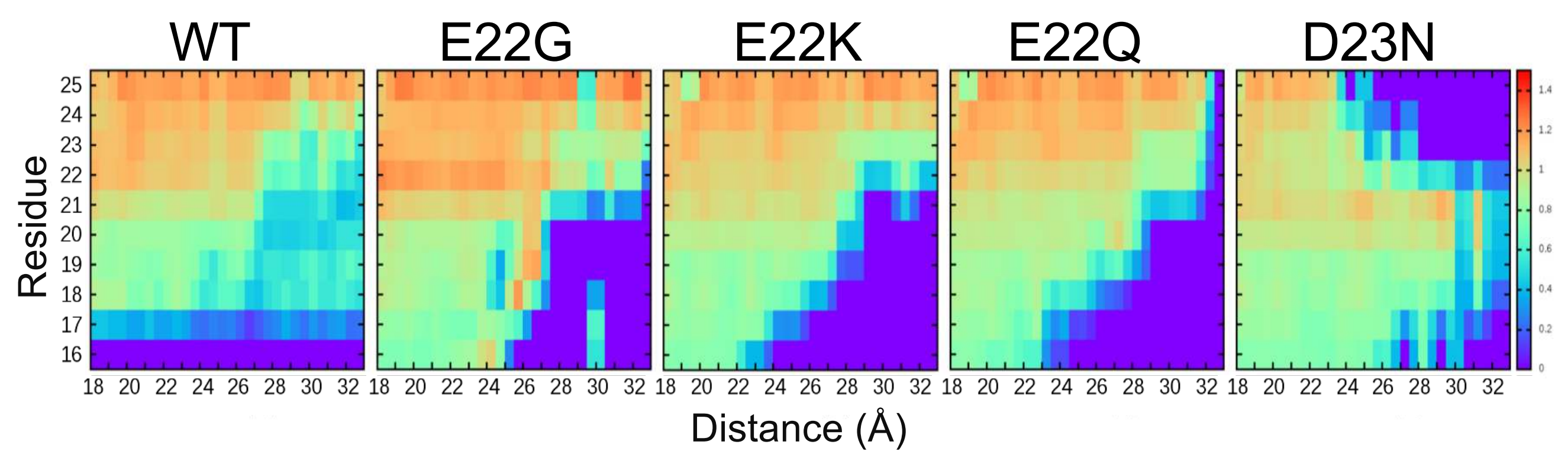

Dipole enhancement as a function of unfolding reveals:

- All mutants have weaker enhancement than WT (backbone $\mathrm{H}$-bonding/dipole alignment less favorable)

- E22[GKQ] enhancements degrade from $\mathrm{N}$-terminus

- D23N enhancement degrades from both termini (weakest $\Delta \mathrm{G}_{\text {fold }}$ of all variants) 


\section{Simulations of Full-Length $A \beta$}

Starting from helical (1IYT)
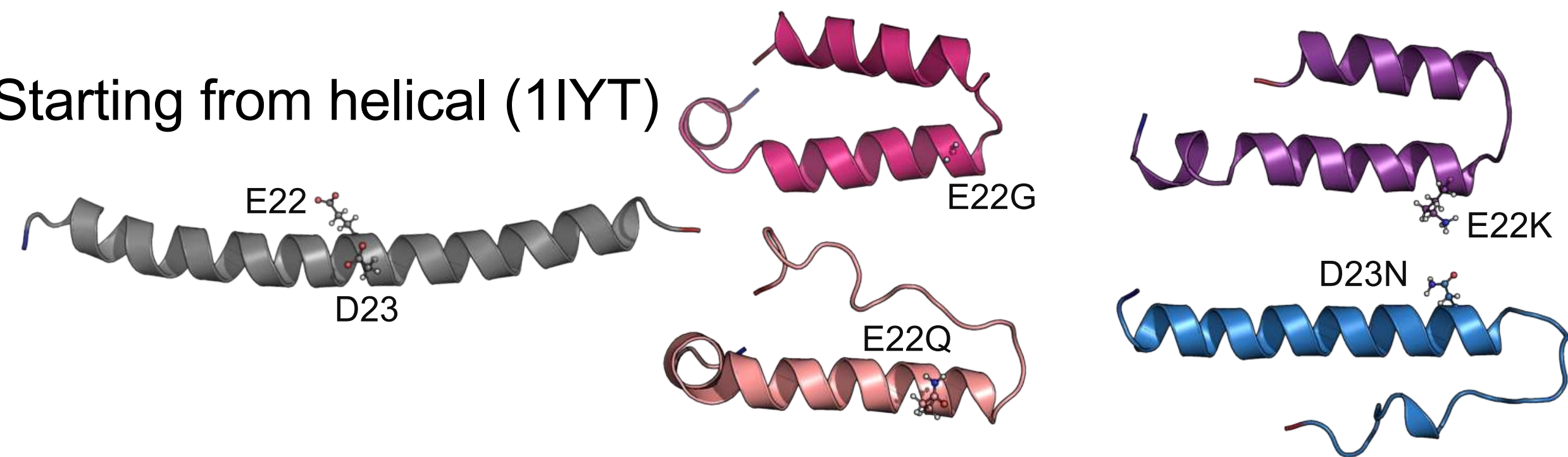

\begin{tabular}{|c|c|c|c|c|}
\cline { 2 - 5 } \multicolumn{1}{c|}{ a-helicity } & \multicolumn{2}{c|}{ C36m } & \multicolumn{2}{c|}{ Drude } \\
\cline { 2 - 5 } \multicolumn{1}{c|}{} & 1IYT & Unfolded & 1IYT & Unfolded \\
\hline WT & $41 \pm 6 \%$ & $21 \pm 2 \%$ & $72 \pm 15 \%$ & $38 \pm 1 \%$ \\
\hline E22G & $40 \pm 10 \%$ & $21 \pm 21 \%$ & $73 \pm 4 \%$ & $31 \pm 22 \%$ \\
\hline E22K & $44 \pm 8 \%$ & $30 \pm 13 \%$ & $76 \pm 4 \%$ & $52 \pm 8 \%$ \\
\hline E22Q & $32 \pm 8 \%$ & $15 \pm 13 \%$ & $61 \pm 6 \%$ & $26 \pm 28 \%$ \\
\hline D23N & $31 \pm 7 \%$ & $24 \pm 2 \%$ & $55 \pm 8 \%$ & $28 \pm 9 \%$ \\
\hline
\end{tabular}




\section{Simulations of Full-Length $A \beta$}

Starting from unfolded
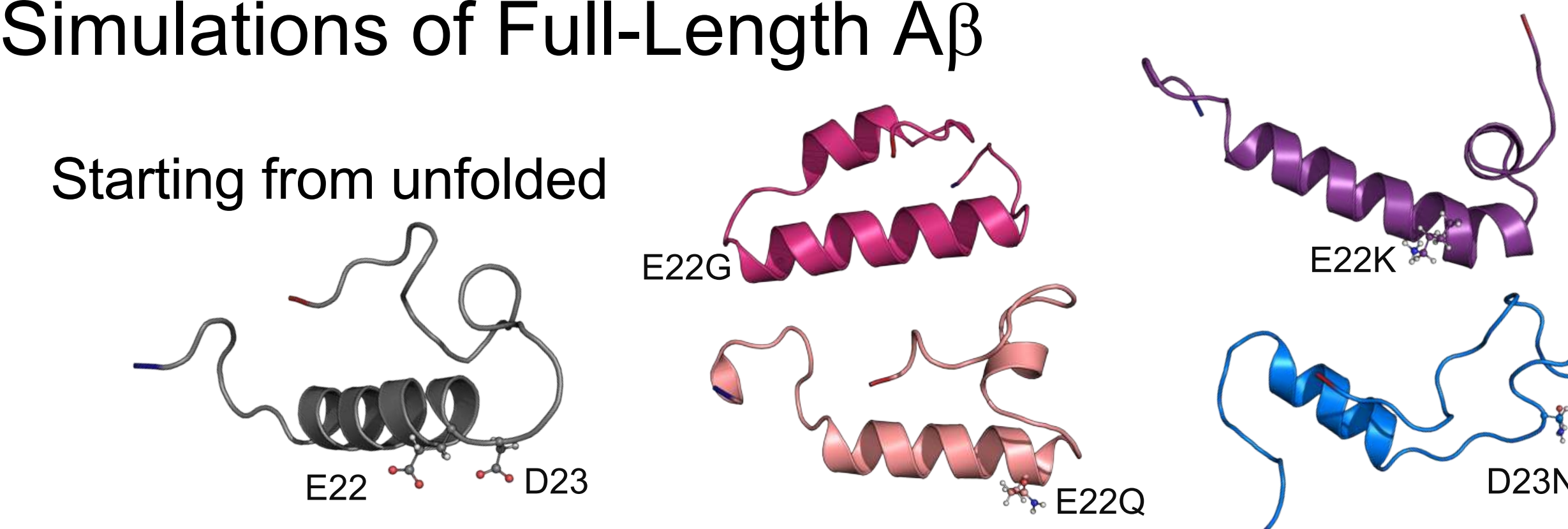

\begin{tabular}{|c|c|c|c|c|}
\cline { 2 - 5 } \multicolumn{1}{c|}{$\boldsymbol{\alpha}$-helicity } & \multicolumn{2}{c|}{$\mathbf{C 3 6 m}$} & \multicolumn{2}{c|}{ Drude } \\
\cline { 2 - 5 } \multicolumn{1}{c|}{} & 1IYT & Unfolded & 1IYT & Unfolded \\
\hline WT & $41 \pm 6 \%$ & $21 \pm 2 \%$ & $72 \pm 15 \%$ & $38 \pm 1 \%$ \\
\hline E22G & $40 \pm 10 \%$ & $21 \pm 21 \%$ & $73 \pm 4 \%$ & $31 \pm 22 \%$ \\
\hline E22K & $44 \pm 8 \%$ & $30 \pm 13 \%$ & $76 \pm 4 \%$ & $52 \pm 8 \%$ \\
\hline E22Q & $32 \pm 8 \%$ & $15 \pm 13 \%$ & $61 \pm 6 \%$ & $26 \pm 28 \%$ \\
\hline D23N & $31 \pm 7 \%$ & $24 \pm 2 \%$ & $55 \pm 8 \%$ & $28 \pm 9 \%$ \\
\hline
\end{tabular}




\section{Conformational Ensembles of Full-Length $A \beta$}

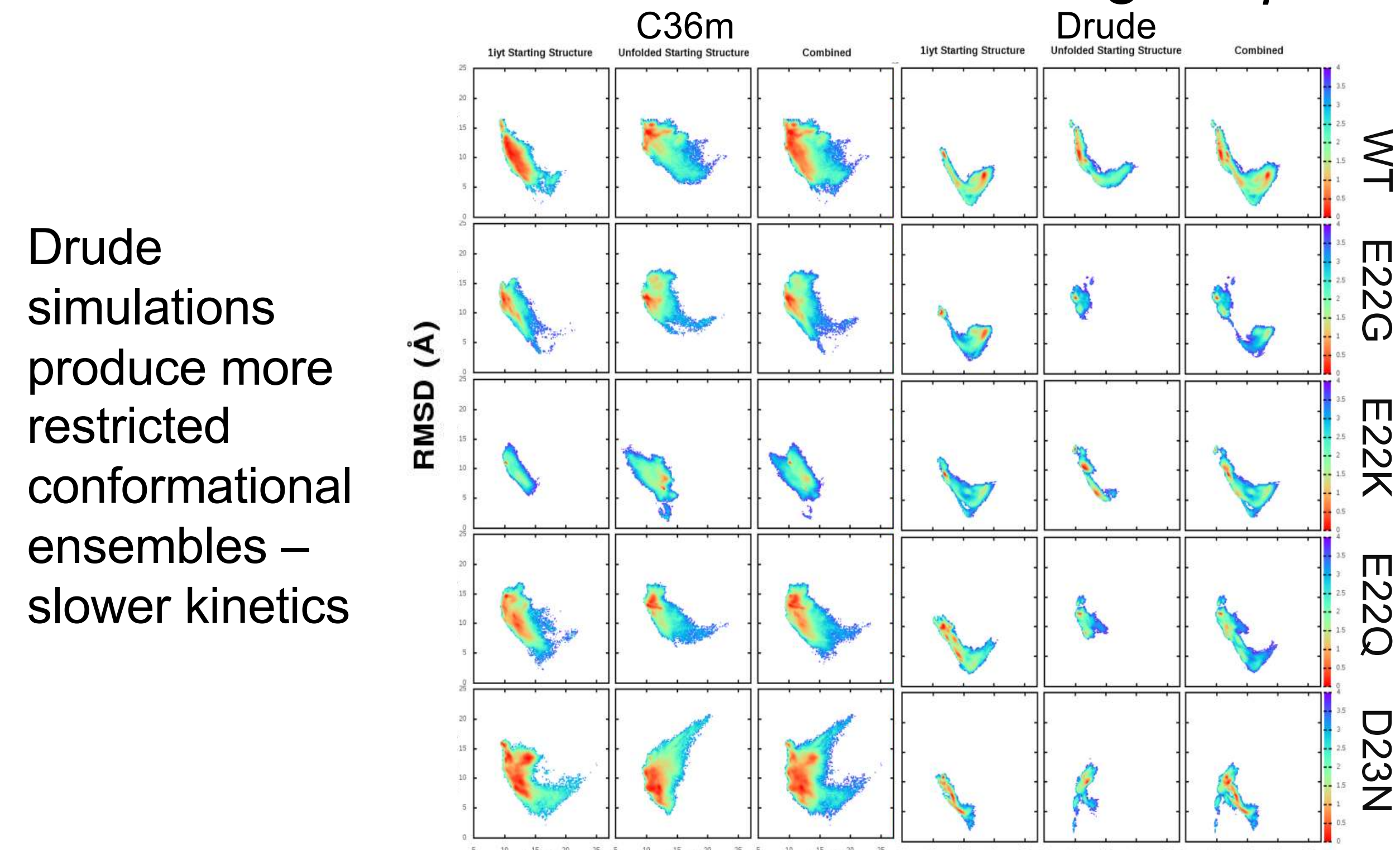

$\operatorname{Rg}(\AA)$ 


\section{Conclusions and Future Directions}

- E22 mutants of $A \beta$ have similar $\Delta \mathrm{G}_{\text {fold }}$ to $\mathrm{WT}$ but dipole enhancement suggests $\mathrm{H}$-bonding has different strength

- D23N mutant has weakest $\Delta \mathrm{G}_{\text {fold }}$ and dipole enhancements are degraded from both termini

- Currently computing PMF at different temperatures to produce van't Hoff plots for $\Delta \mathrm{H}$ and $\Delta \mathrm{S}$

- Are unfolding pathways driven by different forces in different mutants?

- Conformational change is slower (more realistic) with Drude FF so starting from fully $\alpha$-helical $A \beta$ is unreasonable

- More simulations ongoing starting from different disordered conformers 


\section{Acknowledgments}

MacKerell Lab @ UMB

Alex MacKerell

Jing Huang

Alexey Savelyev

NIH)

National Institutes of Health

Turning Discovery Into Health

F32GM109632

R35GM133754

Health Resources in Action Advancing Public Health and Medical Research

Thomas F. and Kate Miller Jeffress Memorial Trust

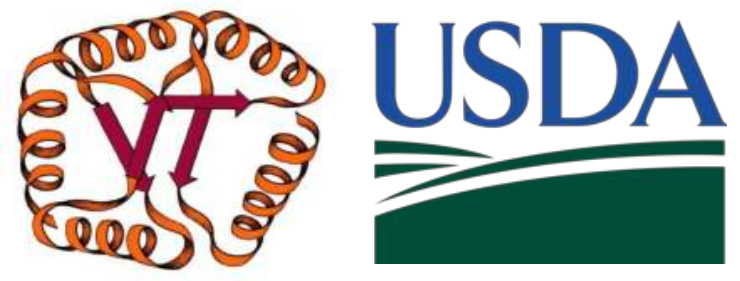

Lemkul Lab @ VT

Alexa Salsbury

Darcy Davidson

Brian Ratnasinghe Julia Montgomery

Tanner Dean

Alexsandra Corrigan

Jesse Janoski

Danielle Porier

Joshua Kraus

Karlie Wysong

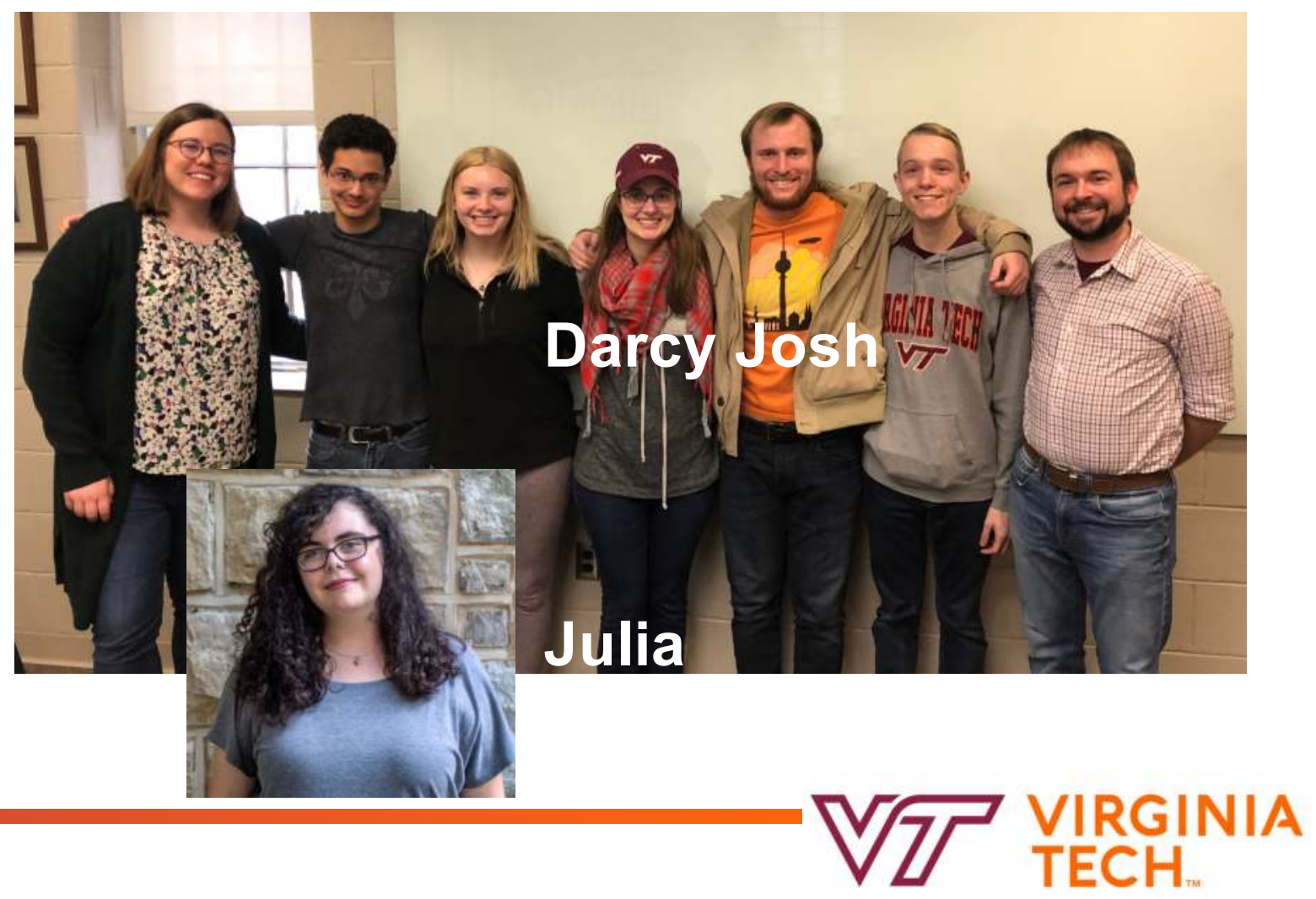

